\title{
Challenges of Toxicity Management in Immuno-Oncology
}

\author{
Presented by Stephanie Andrews, MS, ANP-BC
}

\section{Abstract}

Immunotherapies are conveying unprecedented efficacy in some tumor types, but with this success comes challenges in managing toxicities that are distinct from those of cytotoxic agents. Although most immune-related adverse events can be ameliorated by temporarily withholding the drug and administering steroids, grade 3 to 4 toxicities can be challenging and some adverse effects can be long-lasting. NCCN has developed an immunotherapy teaching and monitoring tool that can help in evaluating and managing these autoimmune-mediated inflammatory conditions, which can affect virtually all organ systems.

J Natl Compr Canc Netw 2017;15(5.5):706-708

Adverse events (AEs) related to immunotherapies are, in general, easily managed, but occasionally can be fatal. Therefore, they should be evaluated promptly and managed appropriately and without delay, said Stephanie Andrews, MS, ANP-BC, Oncology Nurse Practitioner, Moffitt Cancer Center.

Autoimmunity is recognized as an effect of prolonged T-cell activation via inhibition of either CTLA4 or the programmed death protein receptor (PD-1) and its ligand (PD-L1). "We need to balance the efficacy of immunotherapy with a healthy respect for the power of T-cell activation," Ms. Andrews said.

Immune-related AEs (irAEs) include a variety of inflammatory conditions-primarily dermatitis, enterocolitis, hepatitis, endocrinopathies, nephritis, and pneumonitis-but they may affect virtually any organ system. Most irAEs occur within the first 6 to 12 weeks of therapy, but they can also emerge "days, weeks, or months later, or even after discontinuation of therapy," said Ms. Andrews. "Most are easily reversible. However, endocrinopathies may or may not be permanent."

Skin toxicities are the most common irAE seen with checkpoint inhibitors. Dermatitis and colitis occur earlier in the treatment course; dermatitis has the highest

Presented by Stephanie Andrews, MS, ANP-BC, Moffitt Cancer Center, Tampa, Florida.

Ms. Andrews has disclosed that she has served on a speakers' bureau for Genentech, Inc.

Correspondence: Stephanie Andrews, MS, ANP-BC, Moffitt Cancer

Center, 12902 Magnolia Drive, MCC IHM, Tampa, FL 33612.

E-mail: stephanie.andrews@moffitt.org incidence, followed by colitis. Less frequent events are endocrinopathies and hepatic, pulmonary, and renal toxicities, which tend to occur later in the treatment course. The emergence of symptoms related to these conditions should raise suspicion of an irAE; however, clinicians should also consider the possibility of a noninflammatory condition or disease progression.

"Immune-related toxicities may be fatal, and delaying adequate care could lead to poor prognosis," Ms. Andrews emphasized. She offered the slogan "Keep Calm and Slow Down" as a reasonable approach if irAEs flare during a steroid taper, meaning clinicians should re-escalate the taper and then slow it down. After ruling out noninflammatory causes of the symptom, systemic steroids should be used upfront and aggressively, with slow steroid taper, she advised.

\section{Clinical Teaching and Monitoring Tools}

"The education of patients and staff is key for prompt identification of irAEs," she said. To this end, NCCN has developed an immunotherapy teaching and monitoring tool that is available on NCCN.org. ${ }^{1}$ This document includes not only checkpoint inhibitors but also 22 drugs, mostly monoclonal antibodies. Separate sections of the tool are directed to clinicians and patients, with drug-related AEs and symptoms described. It also includes a checklist of symptoms unique to each drug, in the form of a questionnaire that patients can answer before their office visit (Table 1). Symptoms should be 
regularly assessed and education about them reinforced, she said.

Clinicians can also find guidance for managing immune-related toxicities related to checkpoint inhibitors in the NCCN Clinical Practice Guidelines in Oncology (NCCN Guidelines) for Melanoma (Version 1.2017). Melanoma is the tumor type in which perhaps the most experience has been gained with checkpoint inhibitors. Due to the rapid pace of advances in the treatment of patients with cancer, NCCN and ASCO are collaborating to facilitate ongoing clinical guidance on the management of immunotherapy toxicities, with guidelines anticipated later in 2017.

\section{Management of Dermatitis}

Management of toxicities associated with immune checkpoint inhibitors depends on the grade of symptoms. "Grading of symptoms is key to their appropriate management," Ms. Andrews emphasized. Systemic steroids are typically the first line of pharmacologic treatment for most immune-related inflammatory AEs.

Skin toxicity is a common irAE. It can be managed according to the most recent NCCN Guidelines for Melanoma; these are are generally consistent across the checkpoint inhibitors. Prescribing information for ipilimumab states that symptomatic treatment is acceptable for grade 1 toxicity; withholding the drug is recommended for grade 2; and discontinuation of the checkpoint inhibitor and treatment with systemic steroids is appropriate for grades 3 and 4 . According to the package insert, nivolumab should be withheld for grade 3 skin toxicity and resumed on resolution to grade 0 to 1 ; permanent discontinuation is recommended for grade 4 skin rash. Prednisone at 1 to $2 \mathrm{mg} / \mathrm{kg} / \mathrm{d}$, or its equivalent, followed by taper, is also recommended.

The recommendations are similar for pembrolizumab, with the label stating that other systemic immunosuppressants can be considered if grade 3 to 4 dermatitis is not controlled with corticosteroids. This strategy is also advisable for steroid-refractory toxicity stemming from any of the checkpoint inhibitors. Dose reductions are not recommended; instead the drug should be withheld or discontinued.

In general, steroids should be tapered slowly, over at least 4 weeks. Because steroids themselves also have adverse effects, prophylaxis against gastrointestinal problems and opportunistic infection is necessary. Steroid administration for managing irAEs does not affect treatment outcome, ${ }^{2,3}$ Ms. Andrews assured clinicians, but serum glucose should be monitored.

\section{Management of Endocrinopathies, Colitis, and More}

The symptoms of immune-mediated endocrinopathies are vague, and the differential diagnosis can

\begin{tabular}{|l|l|}
\hline \multicolumn{2}{|c|}{ Table 1. NCCN Immunotherapy Teaching/Monitoring Tool ${ }^{1}$} \\
\hline \multicolumn{1}{|c|}{ Pembrolizumab: Patient Checklist } & Yes/No \\
\hline Side Effect & \\
\hline Digestive System & \\
Are you experiencing any diarrhea or an increase in bowel movements? & \\
Are you experiencing any nausea or vomiting? & \\
Do you have abdominal pain or tenderness? & \\
Have your stools changed in color? (eg, bloody, with mucus, dark, and tarry) & \\
Skin & \\
\hline Is your skin dry or itchy? & \\
Do you have a new or worsening rash? Is your skin peeling? & \\
Do you have painful blisters on your body or inside your mouth? & \\
Immune System & \\
Do you have any flu-like symptoms? (eg, fever $>100.4^{\circ} \mathrm{F}\left(38^{\circ} \mathrm{C}\right)$, headache, cough, body aches) & \\
Are you experiencing any redness, pain, or swelling at the infusion site?" & \\
Have you noticed swelling in your face or mouth? &
\end{tabular}

The NCCN Immunotherapy Teaching/Monitoring Tool. ๑ 2017 National Comprehensive Cancer Network, Inc. Available at: NCCN.org. 
Andrews

\begin{tabular}{|c|c|c|}
\hline Ipilimumab ${ }^{1, *}$ & $\begin{array}{l}\text { - Withhold dose } \\
\text { - Resume if resolves to grade } 0-1 \text { and receiving }<7.5 \mathrm{mg} / \mathrm{d} \text { prednisone or equivalent } \\
\text { - Permanently discontinue ipilimumab if: } \\
\text { o Lasts } \geq 6 \text { weeks } \\
\text { o Unable to reduce steroid dose to }<7.5 \mathrm{mg} / \mathrm{d} \text { prednisone or equivalent }\end{array}$ & $\begin{array}{l}\text { - Permanently discontinue ipilimumab } \\
\text { - Systemic corticosteroids: } 1-2 \mathrm{mg} / \mathrm{kg} / \mathrm{d} \text { prednisone } \\
\text { or equivalent }\end{array}$ \\
\hline Nivolumab ${ }^{2,+}$ & $\begin{array}{l}\text { - Withhold dose } \\
\text { - Resume if resolves to grade } 0-1 \\
\text { - Corticosteroids: } 1-2 \mathrm{mg} / \mathrm{kg} / \mathrm{d} \text { prednisone or equivalent, followed by taper }\end{array}$ & $\begin{array}{l}\text { - Permanently discontinue nivolumab } \\
\text { - Corticosteroids: } 1-2 \mathrm{mg} / \mathrm{kg} / \mathrm{d} \text { prednisone or } \\
\text { equivalent, followed by taper }\end{array}$ \\
\hline Pembrolizumab ${ }^{3,4}$ & $\begin{array}{l}\text { - Withhold dose } \\
\text { - Corticosteroids: } 1-2 \mathrm{mg} / \mathrm{kg} / \mathrm{d} \text { prednisone or equivalent, followed by taper } \\
\text { - Resume if resolves to grade } 0-1 \\
\text { - Permanently discontinue pembrolizumab if: } \\
\text { o Recurs } \\
\text { o Unable to reduce steroid dose to } \leq 10 \mathrm{mg} / \mathrm{d} \text { prednisone or equivalent within } 12 \\
\text { weeks } \\
\text { o Does not recover to grade } 0-1 \text { within } 12 \text { weeks of last dose of pembrolizumab }\end{array}$ & $\begin{array}{l}\text { - Permanently discontinue pembrolizumab } \\
\text { - Corticosteroids: } 1-2 \mathrm{mg} / \mathrm{kg} / \mathrm{d} \text { prednisone or } \\
\text { equivalent, followed by a taper }\end{array}$ \\
\hline Atezolizumab ${ }^{4}$ & - Withhold for moderate pneumonitis & $\begin{array}{l}\text { - Permanently discontinue atezolizumab for severe } \\
\text { or life-threatening pneumonitis }\end{array}$ \\
\hline
\end{tabular}

*No specific recommendations for pneumonitis.

tMonitor patients for signs with radiographic imaging and for symptoms of pneumonitis.

${ }^{\ddagger}$ Monitor patients for signs and symptoms of pneumonitis. Evaluate patients with suspected pneumonitis with radiographic imaging

1. Prescribing information: Ipilimumab. Oct 2015. Available at: http://bit.ly/1Rw0nLP

2. Prescribing information: Nivolumab. Feb 2017. Available at: http://bit.ly/1V77FcW

3. Prescribing information: Pembrolizumab. Oct 2016. Available at: http://bit.ly/1NsxJMs

4. Prescribing information: Atezolizumab. Oct 2016. Available at: http://bit.ly/2m9pb5W

be quite extensive. Symptoms can include increased hunger or thirst; changes in weight; and feeling more tired, warmer, or colder than usual; persistent or unusual headache; changes in mood or behavior; hair loss; deeper voice; and constipation. For hypothyroidism, thyroid hormone replacement therapy can be given as needed, without adjusting the dose of the checkpoint inhibitor. The exception is grade 3 to 4 hypothyroidism, which necessitates withholding the drug. Nivolumab should be withheld for grade 2 adrenal insufficiency, and permanently discontinued for grade 3 to 4 toxicity. With pembrolizumab and atezolizumab, grade 3 to 4 adrenal insufficiency also dictates withholding the drug.

The management of diarrhea and colitis differs slightly depending on the drug. Generally, grade 2 colitis (4-6 stools per day over baseline, pain, blood, or mucus) prompts concern. Clinicians should consult the NCCN Guidelines for specific information, including the timing and dose of steroid initiation, resumption of the drug, and permanent discontinuation of the drug.

The management of pneumonitis, a potentially serious irAE associated with checkpoint inhibitors, is shown in Table 2. The NCCN Guidelines recommend infliximab for steroid-refractory colitis and mycophenolate for steroid-refractory hepatitis.

Although underlying autoimmune disorders are generally a contraindication to the use of checkpoint inhibitors, some data suggest that selected patients with these disorders might be effectively and safety treated..$^{4,5}$ At this time, however, this is not recommended.

\section{References}

1. National Comprehensive Cancer Network (NCCN). Immunotherapy Teaching/Monitoring Tool. Available at https://www.nccn.org/ immunotherapy-tool/pdf/NCCN_Immunotherapy_Teaching _ Monitoring_Tool.pdf. Accessed March 25, 2017.

2. Horvat TZ, Adel NG, Dang TO, et al. Immune-related adverse events, need for systemic immunosuppression, and effects on survival and time to treatment failure in patients with melanoma treated with ipilimumab at Memorial Sloan Kettering Cancer Center. J Clin Oncol 2015;33:3193-3198.

3. Weber JS, Antonia SJ, Topalian SL, et al. Safety profile of nivolumab in patients with advanced melanoma: a pooled analysis [abstract]. J Clin Oncol 2015;33(Suppl):Abstract 9018.

4. Johnson DB, Sullivan RJ, Ott PA, et al. Ipilimumab therapy in patients with advanced melanoma and preexisting autoimmune disorders. JAMA Oncol 2016;2:234-240.

5. Menzies AM, Johnson DB, Ramanujam S, et al. Anti-PD-1 therapy in patients with advanced melanoma and preexisting autoimmune disorders or major toxicity with ipilimumab [published online ahead of print September 29, 2016]. Ann Oncol, doi: 10.1093/annonc/mdw443. 